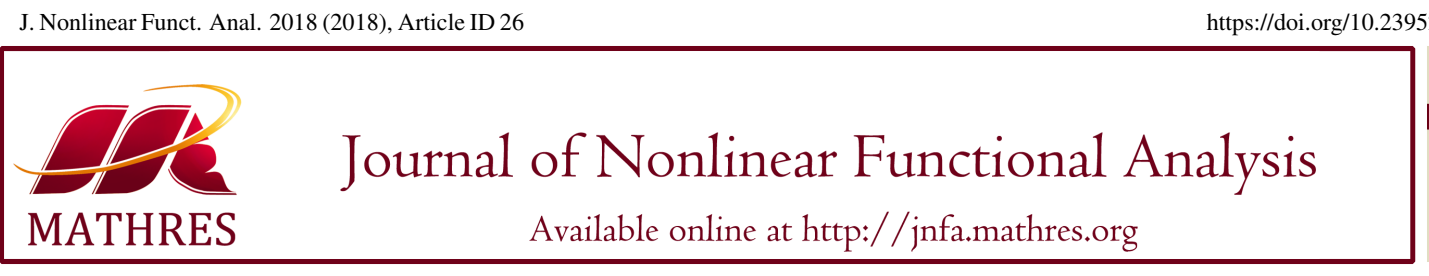

https://doi.org/10.23952/jnfa.2018.26

\title{
A SPLITTING ALGORITHM IN A UNIFORMLY CONVEX AND 2-UNIFORMLY SMOOTH BANACH SPACE
}

\author{
HECAI YUAN
}

School of Mathematics and Statistics, North China University of Water Resources and Electric Power, Henan, China

\begin{abstract}
In this paper, a splitting algorithm is investigated for zero and fixed points of nonlinear operators. A weak convergence theorem is established in a uniformly convex and 2-uniformly smooth Banach space. An application to an equilibrium problem is provided to support our main results.
\end{abstract}

Keywords. Accretive operator; Equilibrium problem; Fixed point; Resolvent; zero point.

2010 Mathematics Subject Classification. 47H05, 47J25, 47J20.

\section{INTRODUCTION}

Throughout this paper, we always assume that $B$ is a reflexive Banach space. Let $C$ be a nonempty closed and convex subset of $B$ and let $B^{*}$ be the topological dual space of $B$. Let $\operatorname{Proj}_{C}^{B}: B \rightarrow C$ be a mapping. Proj $_{C}^{B}$ is said to be

(1) sunny if for each $x \in C$ and $t \in(0,1)$, we have $\operatorname{Proj}_{C}^{B}\left((1-t) \operatorname{Proj}_{C}^{B} x+t x\right)=\operatorname{Proj}_{C}^{B} x$;

(2) contraction if $\left(\operatorname{Proj}_{C}^{B}\right)^{2}=\operatorname{Proj}_{C}^{B}$;

(3) sunny nonexpansive retractction if $P$ ro $j_{C}^{B}$ is contractive, sunny and nonexpansive.

Let $T: C \rightarrow C$ be a mapping. Throughout this paper, we always use Fix $(T)$ to denote the fixed-point set of $T$. Recall that $T$ is said to be contractive iff there exists a constant $\kappa \in(0,1)$ such that

$$
\|T x-T y\| \leq \kappa\|x-y\|, \quad \forall x, y \in C .
$$

From the celebrated Banach fixed-point theorem, we know that Fix $(T)$ is not empty. The classical iterative method to deal with contractive mappings is the Picard iterative method. Recall that $T$ is said to be nonexpansive iff

$$
\|T x-T y\| \leq\|x-y\|, \quad \forall x, y \in C .
$$

The class of nonexpansive mappings is more general than the class of contractive mappings. For nonexpansve mappings, their fixed-point sets may be empty. Let $B=l^{1}$ : all sequences $\left\{x_{n}\right\}$ such that $\sum\left|x_{n}\right|<\infty$

E-mail address: hsyuanhc@yeah.net.

Received January 29, 2018; Accepted August 13, 2018.

(C)2018 Journal of Nonlinear Functional Analysis 
$\left\|x_{n}\right\|_{1}=\sum\left|x_{n}\right|$. Let $T: l^{1} \rightarrow l^{1}$ be the shift operator: $T x_{n}=\left(0, x_{1}, x_{2}, \cdots\right)$

$$
C:=\left\{\left\{x_{n}\right\}: x_{n} \geq 0,\left\|x_{n}\right\|_{1}=1\right\}
$$

Then $T: C \rightarrow C$ is a nonexpansive mapping without a fixed point. Another example, in the framework of infinite dimensional spaces, is from Alspach [1]. Let $B=L^{1}[0,1],\|f\|_{1}=\int_{0}^{1}|f(t)| d_{t}, C:=\left\{f \in L^{1}[0,1]\right.$ : $\left.\int_{0}^{1} f(x) d x=1,0 \leq f \leq 2\right\}$. Then $C$ is weakly compact and convex.

$$
(T f)(t)=\left\{\begin{array}{l}
\min \{2 f(2 t), 2\}, 0 \leq t \leq \frac{1}{2} \\
\min \{2 f(2 t-1)-2,0\}, \frac{1}{2}<t \leq 1
\end{array}\right.
$$

Then $T: C \rightarrow C$ is a nonexpansive mapping without a fixed point. In 1965, Browder [2] proved that if $C$ is also bounded, closed and convex and $B$ is a uniformly convex, then fixed-point sets of nonexpansive mappings are not empty; see also Kirk [3]. Mann-like iterative methods are popular and efficient to deal with fixed points of nonexpansive mappings; see $[4,5,6,7,8]$ and the references therein.

Next, we denote the value of $x^{*} \in B^{*}$ at $x \in B$ by $\left\langle x, x^{*}\right\rangle$. For a sequence $\left\{x_{n}\right\}$ in $B$, we denote strong convergence of $\left\{x_{n}\right\}$ to $x \in B$ by $x_{n} \rightarrow x$ and weak convergence by $x_{n} \rightarrow x$.

Let $q$ be a given real number with $q>1$. The generalized duality mapping $J_{q}: B \rightarrow 2^{B^{*}}$ is defined by

$$
J_{q}(x):=\left\{x^{*} \in B^{*}:\left\langle x^{*}, x\right\rangle=\|x\|^{q},\left\|x^{*}\right\|=\|x\|^{q-1}\right\}, \forall x \in B .
$$

In particular, $J_{2}$ is called the normalized duality mapping. Next, we use $J$ to denote the normalized duality mapping. It is known that

$$
J_{q}(x)=\|x\|^{q-2} J(x) .
$$

It is known that normalized duality mapping $J$ has the following properties [9]:

(1) if $E$ is reflexive, then $J$ is surjective;

(2) if $\mathrm{E}$ is strictly convex, then $J$ is one-to-one and $\left\langle x-y, x^{*}-y^{*}\right\rangle>0$, where $x^{*} \in J(x)$ and $y^{*} \in J(y)$, holds for all $x \neq y$;

(3) if $\mathrm{E}$ is smooth, then $J$ is single-valued;

(4) if $\mathrm{E}$ is uniformly smooth, then $J$ is uniformly norm-to-norm continuous on each bounded subset of $B$.

From now on, we use $j$ to denote the single-valued normalized duality mapping.

Recall that $T$ is said to be a $\kappa$-strict pseudocontraction iff there exist a constant $\kappa \in(0,1)$ and $j(x-y) \in$ $J(x-y)$ such that

$$
\langle T x-T y, j(x-y)\rangle \leq\|x-y\|^{2}-\kappa\|(I-T) x-(I-T) y\|^{2}, \quad \forall x, y \in C .
$$

Rewriting the above inequality, we arrive at

$$
\langle(I-T) x-(I-T) y, j(x-y)\rangle \geq \kappa\|(I-T) x-(I-T) y\|^{2}, \quad \forall x, y \in C .
$$

The class of strict pseudocontractions was introduced by Browder and Petryshyn [10] in a real Hilbert space. Recall that a mapping $A: C \rightarrow B$ is said to be accretive iff there exists $j(x-y) \in J(x-y)$ such that

$$
\langle A x-A y, j(x-y)\rangle \geq 0, \quad \forall x, y \in C .
$$


Recall that a mapping $A: C \rightarrow B$ is said to be inverse-strongly accretive iff there exist $j(x-y) \in J(x-y)$ and a constant $\alpha>0$ such that

$$
\langle A x-A y, j(x-y)\rangle \geq \alpha\|A x-A y\|^{2}, \quad \forall x, y \in C .
$$

Let $M: \operatorname{Dom}(M) \subset B \rightarrow 2^{B}$ be a set-valued operator with domain $\operatorname{Dom}(M)=\{z \in B: M z \neq \emptyset\}$ and range $\operatorname{Ran}(M)=\cup\{M z: z \in \operatorname{Dom}(M)\}$. Recall that $M$ is said to be accretive iff, for all $\lambda>0$ and for each $x, y \in \operatorname{Dom}(M)$,

$$
\left\|\lambda\left(x^{\prime}-y^{\prime}\right)+(x-y)\right\| \geq\|x-y\|, \quad \forall x^{\prime} \in M x, y^{\prime} \in M y .
$$

The accretive operators were introduced independently in 1967 by Browder [11] and Kato [12]. From Kato [12], $A$ is accretive iff, for $x, y \in \operatorname{Dom}(A)$, there exists $j(x-y) \in J(x-y)$ such that

$$
\langle u-v, j(x-y)\rangle \geq 0,
$$

where $u \in M x$ and $v \in M y$. An accretive operator $M$ is said to be $m$-accretive if $\operatorname{Ran}(I+\lambda A)=E$ for all $\lambda>0$. In a Hilbert space, it is maximal monotone.

In this paper, we consider the following problem: finding a point $x$, which is both a fixed point of a strict pseudocontraction $T$ and a zero point of the sum of an $m$-accretive mapping $M$ and inverse-strongly accretive mapping $S$, that is,

$$
x \in \operatorname{Fix}(T) \cap(S+M)^{-1}(0) .
$$

This problem includes fixed point problem and operator splitting problems as special cases. In the framework of Hilbert spaces, the accretivity coincides the monotonicity. Recently, Iterative methods have been introduced and investigated for solving the above problem in Hilbert spaces; see [13, 14, 15, 16] and the references therein. In the framework of Banach spaces, metric projections and resolvents may not be nonexpansive. There are relatively few results in the framework of Banach spaces, we refer the reader to $[17,18,19,21,22]$ and the references therein. The aim of this paper is to present a new weakly convergent method in a uniformly convex and 2-uniformly smooth Banach space. The rest of this paper is organized as follows. In Section 2, necessary preliminaries are provided. In Section 3, the weakly convergent method is presented and an application is also provided.

\section{PRELIMINARIES}

Recall that a Banach space $B$ is said to be uniformly convex if for each $\varepsilon>0$ there is a $\delta>0$ such that, $x, y \in B$ with $\|x\| \leq 1,\|y\| \leq 1$ and $\|x-y\| \geq \varepsilon,\|x+y\| \leq 2(1-\delta)$ holds.

Let $N_{B}=\{x \in B:\|x\|=1\}$. The norm of $B$ is said to be Gâteaux differentiable if the limit $\lim _{t \rightarrow 0}\left(\| \frac{x}{t}+\right.$ $\left.y\|-\| \frac{x}{t} \|\right)$ exists for each $x, y \in N_{B}$. In this case, $B$ is said to be smooth. The norm of $B$ is said to be uniformly Gâteaux differentiable if for each $y \in N_{B}$, the limit is attained uniformly for all $x \in N_{B}$. The norm of $B$ is said to be Fréchet differentiable if for each $x \in N_{B}$, the limit is attained uniformly for all $y \in N_{B}$. The norm of $B$ is said to be uniformly Fréchet differentiable if the limit is attained uniformly for all $x, y \in N_{B}$.

Recall that a mapping $T: C \rightarrow C$ is said to be closed at $y$ if $x_{n} \rightarrow x$ and $T x_{n} \rightarrow y$ imply that $T x=y$. Recall that $T: C \rightarrow C$ is said to be demiclosed at $y$ if $x_{n} \rightarrow x$ and $T x_{n} \rightarrow y$ imply that $T x=y$.

Recall that a Banach space $B$ is said to have the Kadec-Klee property if a sequence $x_{n} \rightarrow x$ and $\left\|x_{n}\right\| \rightarrow\|x\|$, then $x_{n} \rightarrow x$. It is known that if $B$ is uniformly convex, then it has the Kadec-Klee property; see [23] for more details. 
Next, we list some necessary lemmas.

Lemma 2.1 ([24]). Let $C$ be a nonempty closed convex and bounded subset of a real uniformly convex Banach space $B$. Then there is a strictly increasing and continuous convex function $\psi:[0, \infty) \rightarrow[0, \infty)$ with $\varphi(0)=0$ such that, for each nonexpansive mapping $T$ on $C, \forall x, y \in C$ and $\forall t \in[0,1]$,

$$
\psi^{-1}(\|y-x\|-\|T y-T x\|) \geq\|(t T x+(1-t) T y)-T(t x+(1-t) y)\| .
$$

Lemma 2.2 ([9]). A Banach space $E$ is uniformly convex if and only if there exists a continuous strictly increasing convex function $\varphi:[0, \infty) \rightarrow[0, \infty)$ with $\varphi(0)=0$ such that

$$
\|a x+(1-a) y\|^{2} \leq(1-a)\|y\|^{2}+a\|x\|^{2}-(1-a) a \varphi(\|y-x\|),
$$

for all $x, y \in\{x \in B:\|x\| \leq r\}$, where $r>0$ is some real number, and $a \in[0,1]$.

Lemma 2.3 ([9]). Let $B$ be a real 2-uniformly smooth Banach space. Then the following inequality holds: $\|x+y\|^{2}-\|x\|^{2} \leq 2\langle y, j(x+y)\rangle, \forall x, y \in B$, and $\|x+y\|^{2}-\|x\|^{2} \leq 2\langle y, j(x)\rangle+2\|\Upsilon y\|^{2}, \forall x, y \in B$, where $\Upsilon$ is the smooth constant.

Lemma 2.4 ([25]). Let $C$ be a nonempty closed convex subset of a real uniformly convex Banach space $B$. Let $T: C \rightarrow C$ be a strict pseudocontraction. Then $I-T$ is demiclosed at the origin.

Lemma 2.5 ([10]). Let B be a real uniformly convex Banach space. Let $B^{*}$ the dual space of $B$ such that it has the Kadec-Klee property. Suppose that $\left\{x_{n}\right\}$ is a bounded sequence such that $\lim _{n \rightarrow \infty} \|(1-a) p_{1}+$ $a x_{n}-p_{2} \|$ exists for all $a \in[0,1]$ and $p_{1}, p_{2} \in \omega_{w}\left(x_{n}\right)$, where $\omega_{w}\left(x_{n}\right):=\left\{x: \exists x_{n_{i}} \rightarrow x\right\}$ denotes the weak $\omega$-limit set of $\left\{x_{n}\right\}$ Then $\omega_{w}\left(x_{n}\right)$ is a singleton.

Lemma 2.6 ([10]). Let $B$ be a real Banach space and let $C$ be a nonempty closed and convex subset of $B$. Let $S: C \rightarrow B$ be a single valued operator and let $M: B \rightarrow 2^{B}$ be an m-accretive operator. Then

$$
\operatorname{Fix}\left((I+\lambda M)^{-1}(I-\lambda S)\right)=(S+M)^{-1}(0),
$$

where $\lambda$ is any positive real number.

\section{MAIN RESULTS}

Theorem 3.1. Let $B$ be a 2-uniformly smooth (with smooth constant $\Upsilon$ ) and uniformly convex Banach space. Let $C$ be a nonempty closed convex subset of $B$ and let $P$ ro $j_{C}^{B}$ be the sunny nonexpansive retractction from $B$ onto $C$. Let $S: C \rightarrow E$ be an inverse-strongly accretive operator with the constant $\alpha>0$, $M: \operatorname{Dom}(M) \subset B \rightarrow 2^{B}$ an m-accretive operator and $T: C \rightarrow B$ a $\kappa$-strict pseudocontraction such that $(S+M)^{-1}(0) \cap F i x(T) \neq \emptyset$. Let $\left\{x_{n}\right\}$ be a sequence defined by: $x_{0} \in C$ and

$$
x_{n+1}=\operatorname{Proj}_{C}^{B}\left(\left(1-\alpha_{n}\right)\left(I+\lambda_{n} M\right)^{-1}\left(x_{n}-\lambda_{n} S x_{n}\right)+\alpha_{n}\left(\left(1-\beta_{n}\right) x_{n}+\beta_{n} T x_{n}\right)\right),
$$

$n \geq 0$, where $\left\{\lambda_{n}\right\}$ is a positive number sequence, and $\left\{\alpha_{n}\right\}$ and $\left\{\beta_{n}\right\}$ are real number sequences in $(0,1)$. Assume that $\lambda \leq \lambda_{n} \leq \lambda^{\prime}<\frac{\alpha}{\gamma^{2}}$, where $\lambda$ and $\lambda^{\prime}$ are two constants, $0<\alpha \leq \alpha_{n} \leq \alpha^{\prime}<1$, where $\alpha$ and $\alpha^{\prime}$ are two constants, and $\beta \leq \beta_{n} \leq \frac{\kappa}{\Upsilon^{2}}$, where $\beta>0$ is a constant. Then $\left\{x_{n}\right\}$ converges weakly to some point in Fix $(T) \cap(S+M)^{-1}(0)$. 
Proof. The proof is split into the following three steps.

Step 1. Show that both sequence $\left\{x_{n}\right\}$ and sequence $\left\{y_{n}\right\}$ are bounded.

Since Fix $(T) \cap(S+M)^{-1}(0)$ is assumed to be nonempty, we can fix an element in $\operatorname{Fix}(T) \cap(S+$ $M)^{-1}(0)$, say, $x^{*}$. Since $S$ is an inverse-strongly accretive operator, we have

$$
\left\langle S x-S x^{*}, j(x-y)\right\rangle \geq \alpha\left\|S x-S x^{*}\right\|^{2}, \quad \forall x \in C .
$$

Set $y_{n}=\left(1-\beta_{n}\right) x_{n}+\beta_{n} T x_{n}$ and $\Lambda_{n}=x_{n}-\lambda_{n} S x_{n}$. Using Lemma 2.3, we see that

$$
\begin{aligned}
\left\|\left(x^{*}-\lambda_{n} S x^{*}\right)-\Lambda_{n}\right\|^{2} & =\left\|\left(x^{*}-x_{n}\right)-\lambda_{n}\left(S x^{*}-S x_{n}\right)\right\|^{2} \\
& \leq\left\|x_{n}-x^{*}\right\|^{2}-2 \lambda_{n}\left\langle S x^{*}-S x_{n}, j\left(x^{*}-x_{n}\right)\right\rangle+2 \Upsilon^{2} \lambda_{n}^{2}\left\|S x_{n}-S x^{*}\right\|^{2} \\
& \leq\left\|x_{n}-x^{*}\right\|^{2}-2 \lambda_{n}\left(\alpha-\Upsilon^{2} \lambda_{n}\right)\left\|S x^{*}-S x_{n}\right\|^{2} .
\end{aligned}
$$

This in turn implies that

$$
\left\|\left(x^{*}-\lambda_{n} S x^{*}\right)-\Lambda_{n}\right\| \leq\left\|x_{n}-x^{*}\right\|
$$

Note that

$$
\left\langle T x_{n}-T x^{*}, j\left(x_{n}-x^{*}\right)\right\rangle \leq\left\|x_{n}-x^{*}\right\|-\kappa\left\|(I-T) x_{n}\right\| .
$$

Using Lemma 2.3, we see that

$$
\begin{aligned}
\left\|y_{n}-x^{*}\right\|^{2}= & \left\|\left(1-\beta_{n}\right) x_{n}+\beta_{n} T x_{n}-x^{*}\right\|^{2} \\
= & \left\|x_{n}-x^{*}+\beta_{n}\left(T x_{n}-T x^{*}-\left(x_{n}-x^{*}\right)\right)\right\|^{q} \\
\leq & \left\|x_{n}-x^{*}\right\|^{2}+2 \beta_{n}\left(\left\langle T x_{n}-T x^{*}, j\left(x_{n}-x^{*}\right)\right\rangle-\left\langle\left(x_{n}-x^{*}\right), j\left(x_{n}-x^{*}\right)\right\rangle\right) \\
& +2 \Upsilon^{2} \beta_{n}^{2}\left\|T x_{n}-T x^{*}-\left(x_{n}-x^{*}\right)\right\|^{2} \\
\leq & \left\|x_{n}-x^{*}\right\|^{2}+2 \beta_{n}\left(\left\|x_{n}-x^{*}\right\|^{2}-\kappa\left\|(I-T) x_{n}\right\|^{2}\right)-2 \beta_{n}\left\|x_{n}-x^{*}\right\|^{2} \\
& +2 \Upsilon^{2} \beta_{n}^{2}\left\|T x_{n}-x_{n}\right\|^{2} \\
\leq & \left\|x_{n}-x^{*}\right\|^{2}-2 \beta_{n}\left(\kappa-\Upsilon^{2} \beta_{n}\right)\left\|T x_{n}-x_{n}\right\|^{2} .
\end{aligned}
$$

This shows that $\left\|y_{n}-x^{*}\right\| \leq\left\|x_{n}-x^{*}\right\|$ for each $n \geq 1$. It follows that

$$
\begin{aligned}
\left\|x_{n+1}-x^{*}\right\| & \leq\left\|\left(1-\alpha_{n}\right)\left(I+\lambda_{n} M\right)^{-1}\left(x_{n}-\lambda_{n} S x_{n}\right)+\alpha_{n}\left(\left(1-\beta_{n}\right) x_{n}+\beta_{n} T x_{n}\right)-x^{*}\right\| \\
& \leq \alpha_{n}\left\|x_{n}-x^{*}\right\|+\left(1-\alpha_{n}\right)\left\|\left(I+\lambda_{n} M\right)^{-1}\left(x_{n}-\lambda_{n} S x_{n}\right)-\left(I+\lambda_{n} M\right)^{-1}\left(x^{*}-\lambda_{n} S x^{*}\right)\right\| \\
& \leq \alpha_{n}\left\|x_{n}-x^{*}\right\|+\left(1-\alpha_{n}\right)\left\|\left(x^{*}-\lambda_{n} S x^{*}\right)-\Lambda_{n}\right\| \\
& \leq\left\|x_{n}-x^{*}\right\| .
\end{aligned}
$$

This implies that $\lim _{n \rightarrow \infty}\left\|x_{n}-p\right\|$. Hence, $\left\{x_{n}\right\}$ is a bounded sequence. From (3.3), we see that $\left\{y_{n}\right\}$ is also a bounded sequence. This completes that both sequence $\left\{x_{n}\right\}$ and sequence $\left\{y_{n}\right\}$ are bounded.

Step 2. $\omega_{w}\left(x_{n}\right) \subset(S+M)^{-1}(0) \cap F i x(T)$, where $\omega_{w}\left(x_{n}\right)$ is the weak limit set of $\left\{x_{n}\right\}$.

Set $\xi_{n}=\left(I+\lambda_{n} M\right)^{-1}\left(x_{n}-\lambda_{n} S x_{n}\right)$. Since $M$ is an $m$-accretive operator, we have

$$
\left\langle\frac{x_{n}-(I+\lambda M)^{-1}(I-\lambda S) x_{n}}{\lambda}-\frac{x_{n}-\xi_{n}}{\lambda_{n}}, j\left((I+\lambda M)^{-1}(I-\lambda S) x_{n}-\xi_{n}\right)\right\rangle \geq 0 .
$$


Hence,

$$
\begin{aligned}
& \left\|(I+\lambda M)^{-1}(I-\lambda S) x_{n}-\xi_{n}\right\|^{2} \\
& \leq \frac{\left|\lambda_{n}-\lambda\right|}{\lambda_{n}}\left\langle x_{n}-\xi_{n}, j\left((I+\lambda M)^{-1}(I-\lambda S) x_{n}-\xi_{n}\right)\right\rangle \\
& \leq\left\|(I+\lambda M)^{-1}(I-\lambda S) x_{n}-\xi_{n}\right\|\left\|x_{n}-\xi_{n}\right\| .
\end{aligned}
$$

This shows that

$$
\left\|(I+\lambda M)^{-1}(I-\lambda S) x_{n}-\xi_{n}\right\| \leq\left\|x_{n}-\xi_{n}\right\| .
$$

On the other hand, it follows Lemma 2.2 that

$$
\begin{aligned}
\left\|x_{n+1}-x^{*}\right\|^{2} & \leq\left\|\left(1-\alpha_{n}\right) \xi_{n}+\alpha_{n} y_{n}-x^{*}\right\|^{2} \\
& \leq \alpha_{n}\left\|y_{n}-x^{*}\right\|^{2}+\left(1-\alpha_{n}\right)\left\|\xi_{n}-x^{*}\right\|^{2}-\left(1-\alpha_{n}\right) \alpha_{n} \varphi\left(\left\|\xi_{n}-y_{n}\right\|\right) \\
& \leq \alpha_{n}\left\|x_{n}-x^{*}\right\|^{2}+\left(1-\alpha_{n}\right)\left\|\Lambda_{n}-\left(I-\lambda_{n} S\right) x^{*}\right\|^{2}-\left(1-\alpha_{n}\right) \alpha_{n} \varphi\left(\left\|\xi_{n}-y_{n}\right\|\right) \\
& \leq\left\|x_{n}-x^{*}\right\|^{2}-\left(1-\alpha_{n}\right) \alpha_{n} \varphi\left(\left\|\xi_{n}-y_{n}\right\|\right) .
\end{aligned}
$$

Hence,

$$
\begin{aligned}
\left(1-\alpha_{n}\right) \alpha_{n} \varphi\left(\left\|\xi_{n}-y_{n}\right\|\right) & \leq\left\|x_{n}-x^{*}\right\|^{2}-\left\|x_{n+1}-x^{*}\right\|^{2} \\
& \leq \psi\left(\left\|x_{n}-x^{*}\right\|-\left\|x_{n+1}-x^{*}\right\|\right)
\end{aligned}
$$

where $\psi$ is an appropriate constant. So,

$$
\lim _{n \rightarrow \infty} \varphi\left(\left\|\xi_{n}-y_{n}\right\|\right)=0
$$

Using the property of $\varphi$, we find that $\lim _{n \rightarrow \infty}\left\|\xi_{n}-y_{n}\right\|=0$. Since $\|\cdot\|^{2}$ is a convex function, we also have

$$
\begin{aligned}
\left\|x_{n+1}-x^{*}\right\|^{2} & \leq\left\|\left(1-\alpha_{n}\right) \xi_{n}+\alpha_{n} y_{n}-x^{*}\right\|^{2} \\
& \leq\left(1-\alpha_{n}\right)\left\|\xi_{n}-x^{*}\right\|^{2}+\alpha_{n}\left\|y_{n}-x^{*}\right\|^{2} \\
& \leq\left(1-\alpha_{n}\right)\left\|\xi_{n}-x^{*}\right\|^{2}+\alpha_{n}\left\|x_{n}-x^{*}\right\|^{2} .
\end{aligned}
$$

Using Lemma 2.2, we find that

$$
\begin{aligned}
\left\|\xi_{n}-x^{*}\right\|^{2} \leq & \left\|\frac{\lambda_{n}}{2}\left(\frac{\Lambda_{n}-\xi_{n}}{\lambda_{n}}-\frac{\left(I-\lambda_{n} S\right) x^{*}-x^{*}}{\lambda_{n}}\right)+\left(\xi_{n}-x^{*}\right)\right\|^{2} \\
= & \left\|\frac{1}{2}\left(\Lambda_{n}-\left(I-\lambda_{n} S\right) x^{*}\right)+\frac{1}{2}\left(\xi_{n}-x^{*}\right)\right\|^{2} \\
\leq & \frac{1}{2}\left\|\Lambda_{n}-\left(I-\lambda_{n} S\right) x^{*}\right\|^{2}+\frac{1}{2}\left\|\xi_{n}-x^{*}\right\|^{2} \\
& -\frac{1}{4} \varphi\left(\left\|\left(\xi_{n}-x^{*}\right)-\left(\Lambda_{n}-\left(I-\lambda_{n} S\right) x^{*}\right)\right\|\right) \\
\leq & \left\|\Lambda_{n}-\left(I-\lambda_{n} S\right) x^{*}\right\|^{2}-\frac{1}{4} \varphi\left(\left\|\left(\xi_{n}-x^{*}\right)-\left(\Lambda_{n}-\left(I-\lambda_{n} S\right) x^{*}\right)\right\|\right) \\
\leq & \left\|x_{n}-x^{*}\right\|^{2}-\frac{1}{4} \varphi\left(\left\|\left(\xi_{n}-x^{*}\right)-\left(\Lambda_{n}-\left(I-\lambda_{n} S\right) x^{*}\right)\right\|\right) \\
& -2 \lambda_{n}\left(\alpha-\Upsilon^{2} \lambda_{n}\right)\left\|S x^{*}-S x_{n}\right\|^{2} .
\end{aligned}
$$


Substituting (3.6) and (3.5) yields that

$$
\begin{aligned}
\left\|x_{n+1}-x^{*}\right\|^{2} & \leq\left(1-\alpha_{n}\right)\left\|\xi_{n}-x^{*}\right\|^{2}+\alpha_{n}\left\|x_{n}-x^{*}\right\|^{2} \\
& \leq\left\|x_{n}-x^{*}\right\|^{2}-\left(1-\alpha_{n}\right) \frac{1}{4} \varphi\left(\left\|\left(\xi_{n}-x^{*}\right)-\left(\Lambda_{n}-\left(I-\lambda_{n} S\right) x^{*}\right)\right\|\right) \\
& \left.-2\left(1-\alpha_{n}\right) \lambda_{n}\left(\alpha-\Upsilon^{2} \lambda_{n}\right)\left\|S x^{*}-S x_{n}\right\|^{2}\right) .
\end{aligned}
$$

From (3.7), we have

$$
\begin{aligned}
2\left(1-\alpha_{n}\right) \lambda_{n}\left(\alpha-\Upsilon^{2} \lambda_{n}\right)\left\|S x^{*}-S x_{n}\right\|^{2} & \leq\left\|x_{n}-x^{*}\right\|^{2}-\left\|x_{n+1}-x^{*}\right\|^{2} \\
& \leq \tau\left(\left\|x_{n}-x^{*}\right\|-\left\|x_{n+1}-x^{*}\right\|\right),
\end{aligned}
$$

where $\tau$ is an appropriate constant. This implies that

$$
\lim _{n \rightarrow \infty}\left\|S x_{n}-S x^{*}\right\|=0 .
$$

From (3.7), we also have

$$
\begin{aligned}
\left(1-\alpha_{n}\right) \frac{1}{4} \varphi\left(\left\|\left(\xi_{n}-x^{*}\right)-\left(\Lambda_{n}-\left(I-\lambda_{n} S\right) x^{*}\right)\right\|\right) & \leq\left\|x_{n}-x^{*}\right\|^{2}-\left\|x_{n+1}-x^{*}\right\|^{2} \\
& \leq v\left(\left\|x_{n}-x^{*}\right\|-\left\|x_{n+1}-x^{*}\right\|\right)
\end{aligned}
$$

where $v$ is an appropriate constant. This implies that

$$
\lim _{n \rightarrow \infty} \varphi\left(\left\|\left(\xi_{n}-x^{*}\right)-\left(\Lambda_{n}-\left(I-\lambda_{n} S\right) x^{*}\right)\right\|\right)=0 .
$$

Using the property of $\varphi$, we find that

$$
\lim _{n \rightarrow \infty}\left\|\xi_{n}-x_{n}-\lambda_{n}\left(S x^{*}-S x_{n}\right)\right\|=0 .
$$

In view of

$$
\left\|\xi_{n}-x_{n}\right\| \leq\left\|\xi_{n}-x_{n}-\lambda_{n}\left(S x^{*}-S x_{n}\right)\right\|+\lambda_{n}\left\|S x^{*}-S x_{n}\right\|
$$

we arrive at

$$
\lim _{n \rightarrow \infty}\left\|\xi_{n}-x_{n}\right\|=0
$$

It follows from (3.4) that

$$
\lim _{n \rightarrow \infty}\left\|(I+\lambda M)^{-1}(I-\lambda S) x_{n}-\xi_{n}\right\|=0
$$

Note that

$$
\left\|(I+\lambda M)^{-1}(I-\lambda S) x_{n}-x_{n}\right\| \leq\left\|(I+\lambda M)^{-1}(I-\lambda S) x_{n}-\xi_{n}\right\|+\left\|\xi_{n}-x_{n}\right\|
$$

This shows that

$$
\lim _{n \rightarrow \infty}\left\|(I+\lambda M)^{-1}(I-\lambda S) x_{n}-x_{n}\right\|=0 .
$$

Note that

$$
\begin{aligned}
\left\|x_{n}-T x_{n}\right\| & \leq\left\|x_{n}-\xi_{n}\right\|+\left\|\xi_{n}-y_{n}\right\|+\left\|y_{n}-T x_{n}\right\| \\
& \leq\left\|x_{n}-\xi_{n}\right\|+\left\|\xi_{n}-y_{n}\right\|+\left(1-\beta_{n}\right)\left\|x_{n}-T x_{n}\right\| .
\end{aligned}
$$

This implies that

$$
\lim _{n \rightarrow \infty}\left\|x_{n}-T x_{n}\right\|=0
$$

Since $(I+\lambda M)^{-1}(I-\lambda S)$ has the property of nonexpansivity, we find that $\omega_{w}\left(x_{n}\right) \subset(I+\lambda M)^{-1}(I-\lambda S)$. Since $T$ is a strict pseudocontraction, we find from Lemma 2.4 that $\omega_{w}\left(x_{n}\right) \subset F i x(T)$. This proves that

$$
\omega_{w}\left(x_{n}\right) \subset \operatorname{Fix}(T) \cap(S+M)^{-1}(0) .
$$


Step 3. $\omega_{w}\left(x_{n}\right)$ is a singleton, which completes the whole proof.

Next, putting $T_{n}=\beta_{n} T+\left(1-\beta_{n}\right) I$, we see from Lemma 2.3 that, $\forall x, y \in B$,

$$
\begin{aligned}
\left\|T_{n} x-T_{n} y\right\|^{2} & \leq\|x-y\|^{2}+2 \beta_{n}\langle T x-T y-(x-y), j(x-y)\rangle+2 \Upsilon^{2} \beta_{n}^{2}\|T x-T y-(x-y)\|^{q} \\
& =\|x-y\|^{2}+2 \beta_{n}\langle T x-T y, j(x-y)\rangle-2 \beta_{n}\|x-y\|^{2}+2 \Upsilon^{2} \beta_{n}^{2}\|T x-T y-(x-y)\|^{2} \\
& \leq\|x-y\|^{2}-2 \beta_{n}\left(\kappa-\Upsilon^{2} \beta_{n}\right)\|T x-T y-(x-y)\|^{2} . \\
& \leq\|x-y\|^{2} .
\end{aligned}
$$

This shows that $T_{n}$ is a nonexpansive mapping for each $n \geq 1$. Define mappings $W_{n}: C \rightarrow C$ by

$$
W_{n}:=\operatorname{Proj}_{C}^{B}\left(\alpha_{n} T_{n}+\left(1-\alpha_{n}\right)\left(I+\lambda_{n} M\right)^{-1}\left(I-\lambda_{n} S\right)\right) .
$$

and set

$$
W_{n, m}=W_{n+m-1} W_{n+m-2} \cdots W_{n}, \quad \forall n, m \geq 1 .
$$

It follows that $W_{n, m} x_{n}=x_{n+m}$. Note that

$$
\begin{aligned}
\left\|W_{n} x-W_{n} y\right\|= & \| \operatorname{Proj}_{C}^{B}\left(\alpha_{n} T_{n}+\left(1-\alpha_{n}\right)\left(I+\lambda_{n} M\right)^{-1}\left(I-\lambda_{n} S\right)\right) x \\
& -\operatorname{Proj}_{C}^{B}\left(\alpha_{n} T_{n}+\left(1-\alpha_{n}\right)\left(I+\lambda_{n} M\right)^{-1}\left(I-\lambda_{n} S\right)\right) y \| \\
\leq & \left(1-\alpha_{n}\right)\left\|\left(I-\lambda_{n} S\right) x-\left(I-\lambda_{n} S\right) y\right\|+\alpha_{n}\left\|T_{n} x-T_{n} y\right\| \\
\leq & \|x-y\|, \quad \forall x, y \in C .
\end{aligned}
$$

This shows that each $W_{n}$ has the property of nonexpasivity. For all $t \in[0,1]$ and $n, m \geq 1$, put

$$
b_{n}(t)=\left\|t x_{n}-p_{2}+(1-t) p_{1}\right\|,
$$

and

$$
c_{n, m}=\left\|W_{n, m}\left(t x_{n}+(1-t) p_{1}\right)-\left(t x_{n+m}+(1-t) p_{1}\right)\right\|,
$$

where $p_{1}$ and $p_{2}$ are in $(S+M)^{-1}(0) \cap F i x(T)$. By virtue of Lemma 2.1, one has

$$
\begin{aligned}
c_{n, m} & \leq \psi^{-1}\left(\left\|x_{n}-p_{1}\right\|-\left\|W_{n, m} p_{1}-W_{n, m} x_{n}\right\|\right) \\
& \leq \psi^{-1}\left(\left\|x_{n}-p_{1}\right\|-\left(\left\|p_{1}-x_{n+m}\right\|-\left\|W_{n, m} p_{1}-p_{1}\right\|\right)\right),
\end{aligned}
$$

which shows that $\left\{c_{n, m}\right\}$ converges uniformly to zero as $n \rightarrow \infty$ for all $m \geq 1$. Note that

$$
\begin{aligned}
b_{n+m}(t) & \leq\left\|W_{n, m}\left(t x_{n}+(1-t) p_{1}\right)-p_{2}\right\|+c_{n, m} \\
& \leq\left\|W_{n, m}\left(t x_{n}+(1-t) p_{1}\right)-S^{n, m} p_{2}\right\|+c_{n, m}+\left\|p_{2}-W_{n, m} p_{2}\right\| \\
& \leq b_{n}(t)+c_{n, m}+\left\|p_{2}-W_{n, m} p_{2}\right\|,
\end{aligned}
$$

from which it is not hard to see for each $t \in[0,1]$ that $\lim _{n \rightarrow \infty} b_{n}(t)$ exists. Using Lemma 2.5, we find that $\omega_{w}\left(x_{n}\right)$ is a singleton set. This proves the theorem.

If $T$ is nonexpansive, that is, $\kappa=\frac{1}{2}$, we find the following result immediately.

Corollary 3.2. Let $B$ be a 2-uniformly smooth (with smooth constant $\Upsilon$ ) and uniformly convex Banach space. Let $C$ be a nonempty closed convex subset of $B$ and let $\operatorname{Proj}_{C}^{B}$ be the sunny nonexpansive retractction from $B$ onto $C$. Let $S: C \rightarrow E$ be an inverse-strongly accretive operator with the constant 
$\alpha>0, M: \operatorname{Dom}(M) \subset B \rightarrow 2^{B}$ an m-accretive operator and $T: C \rightarrow B$ a nonexpansive mapping such that $(S+M)^{-1}(0) \cap \operatorname{Fix}(T) \neq \emptyset$. Let $\left\{x_{n}\right\}$ be a sequence defined by: $x_{0} \in C$ and

$$
x_{n+1}=\operatorname{Proj}_{C}^{B}\left(\left(1-\alpha_{n}\right)\left(I+\lambda_{n} M\right)^{-1}\left(x_{n}-\lambda_{n} S x_{n}\right)+\alpha_{n}\left(\left(1-\beta_{n}\right) x_{n}+\beta_{n} T x_{n}\right)\right),
$$

$n \geq 0$, where $\left\{\lambda_{n}\right\}$ is a positive number sequence, and $\left\{\alpha_{n}\right\}$ and $\left\{\beta_{n}\right\}$ are real number sequences in $(0,1)$. Assume that $\lambda \leq \lambda_{n} \leq \lambda^{\prime}<\frac{\alpha}{r^{2}}$, where $\lambda$ and $\lambda^{\prime}$ are two constants, $0<\alpha \leq \alpha_{n} \leq \alpha^{\prime}<1$, where $\alpha$ and $\alpha^{\prime}$ are two constants, and $\beta \leq \beta_{n} \leq \frac{1}{2 \Upsilon^{2}}$, where $\beta>0$ is a constant. Then $\left\{x_{n}\right\}$ converges weakly to some point in Fix $(T) \cap(S+M)^{-1}(0)$.

Furthermore, if $T$ is an identity mapping, we have the following result immediately.

Corollary 3.3. Let $B$ be a 2-uniformly smooth (with smooth constant $\Upsilon$ ) and uniformly convex Banach space. Let $C$ be a nonempty closed convex subset of $B$ and let Pro $j_{C}^{B}$ be the sunny nonexpansive retractction from $B$ onto $C$. Let $S: C \rightarrow E$ be an inverse-strongly accretive operator with the constant $\alpha>0$ and let $M: \operatorname{Dom}(M) \subset B \rightarrow 2^{B}$ be an m-accretive operator such that $(S+M)^{-1}(0) \neq \emptyset$. Let $\left\{x_{n}\right\}$ be a sequence defined by: $x_{0} \in C$ and

$$
x_{n+1}=\operatorname{Proj}_{C}^{B}\left(\left(1-\alpha_{n}\right)\left(I+\lambda_{n} M\right)^{-1}\left(x_{n}-\lambda_{n} S x_{n}\right)+\alpha_{n} x_{n}\right),
$$

$n \geq 0$, where $\left\{\lambda_{n}\right\}$ is a positive number sequence, and $\left\{\alpha_{n}\right\}$ is a real number sequence in $(0,1)$. Assume that $\lambda \leq \lambda_{n} \leq \lambda^{\prime}<\frac{\alpha}{\gamma^{2}}$, where $\lambda$ and $\lambda^{\prime}$ are two constants, $0<\alpha \leq \alpha_{n} \leq \alpha^{\prime}<1$, where $\alpha$ and $\alpha^{\prime}$ are two constants. Then $\left\{x_{n}\right\}$ converges weakly to some point in $(S+M)^{-1}(0)$.

We remark here that a typical example of both uniformly convex and 2-uniformly smooth Banachs is $L^{2}$. In addition, if the domain of $M$ is included in $C$, then $\operatorname{Proj}_{C}^{B}$ can be removed from all the above convergence theorems.

Finally, we give an applications to equilibrium problems in a Hilbert space $H$.

Let $C$ be a nonempty closed and convex subset of $H$. Let $F$ be a bifunction of $C \times C$ into $\mathbb{R}$, where $\mathbb{R}$ denotes the set of real numbers. Recall the following equilibrium problem.

$$
\text { Find } x \in C \text { such that } F(x, y) \geq 0, \quad \forall y \in C \text {. }
$$

To study the equilibrium problem, we may assume that $F$ satisfies the following conditions:

(C1) for each $x, y, z \in C$,

$$
\limsup _{t \downarrow 0} F(t z+(1-t) x, y) \leq F(x, y) ;
$$

(C2) for each $x \in C, y \mapsto F(x, y)$ is convex and weakly lower semi-continuous,

(C3) $F(x, y)+F(y, x) \leq 0$ for all $x, y \in C$;

(C4) $F(x, x)=0$ for all $x \in C$;

If $F(x, y)=\langle A x, y-x\rangle$, where $A: C \rightarrow H$ is some monotone operator, for every $x, y \in C$, we see that the equilibrium problem is reduced to a monotone variational inequality problem.

Lemma 3.4. [28] Let $C$ be a nonempty closed convex subset of $H$ and let $F: C \times C \rightarrow \mathbb{R}$ be a bifunction with conditions (C1)-(C4). Then, for any $r>0$ and $x \in H$, there exists $z \in C$ such that

$$
\langle y-z, z-x\rangle+r F(z, y) \geq 0, \quad \forall y \in C .
$$


Further, define

$$
T_{r} x=\{z \in C:\langle y-z, z-x\rangle+r F(z, y) \geq 0, \quad \forall y \in C\}
$$

for all $r>0$ and $x \in H$. Then

(a) $T_{r}$ is single-valued firmly nonexpansive, i.e.,

$$
\left\|T_{r} x-T_{r} y\right\|^{2} \leq\left\langle T_{r} x-T_{r} y, x-y\right\rangle, \quad \forall x, y \in H ;
$$

(b) $F i x\left(T_{r}\right)=E P(F)$, where $E P(F)$ denotes the solution set of the equilibrium problem, is closed and convex.

Lemma 3.5. [29] Let $C$ be a nonempty closed convex subset of a real Hilbert space $H$. Let $F$ a bifunction from $C \times C$ to $\mathbb{R}$ with conditions $(C 1)-(C 4)$ and let $A_{F}$ be a multivalued mapping on $H$ defined by

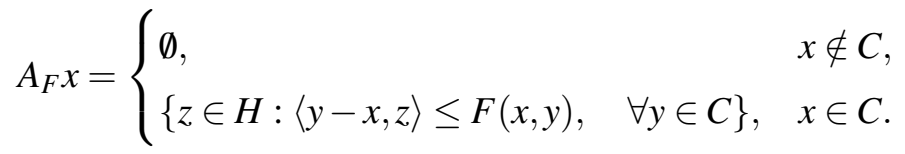

Then $A_{F}$ is a maximal monotone operator with the domain $\operatorname{Dom}\left(A_{F}\right) \subset C, T_{\lambda}=\left(I+\lambda A_{F}\right)^{-1}, \forall \lambda>0$, and $\operatorname{EP}(F)=A_{F}^{-1}(0)$.

With the above tools, we can find the following result immediately.

Theorem 3.6. Let $H$ be a Hilbert space and let $C$ be a nonempty closed convex subset of $H$. Let $T: C \rightarrow B$ be a $\kappa$-strict pseudocontraction. Let $F$ a bifunction from $C \times C$ to $\mathbb{R}$ with conditions $(C 1)-(C 4)$. Assume that $\operatorname{EP}(F) \cap \operatorname{Fix}(T) \neq \emptyset$, where $\operatorname{EP}(F)$ denote the solution set of the equilibrium problem. Let $\left\{x_{n}\right\}$ be a sequence defined by: $x_{0} \in C$ and

$$
x_{n+1}=\left(1-\alpha_{n}\right) z_{n}+\alpha_{n}\left(\left(1-\beta_{n}\right) x_{n}+\beta_{n} T x_{n}\right),
$$

$n \geq 0$, where $z_{n} \in C$ such that

$$
\left\langle u-z_{n}, z_{n}-x_{n}\right\rangle+\lambda_{n} F\left(z_{n}, u\right) \geq 0, \quad \forall u \in C .
$$

$\left\{\lambda_{n}\right\}$ is a positive number sequence, and $\left\{\alpha_{n}\right\}$ and $\left\{\beta_{n}\right\}$ are real number sequences in $(0,1)$. Assume that $\lambda \leq \lambda_{n} \leq \lambda^{\prime}<\frac{\alpha}{\gamma^{2}}$, where $\lambda$ and $\lambda^{\prime}$ are two constants, $0<\alpha \leq \alpha_{n} \leq \alpha^{\prime}<1$, where $\alpha$ and $\alpha^{\prime}$ are two constants, and $\beta \leq \beta_{n} \leq 2 \kappa$, where $\beta>0$ is a constant. Then $\left\{x_{n}\right\}$ converges weakly to some point in $\operatorname{Fix}(T) \cap E P(F)$.

Proof. From Lemma 3.5, we find that the iterative method in Theorem 3.6 can be rewritten as

$$
x_{n+1}=\left(1-\alpha_{n}\right) T_{\lambda_{n}} x_{n}+\alpha_{n}\left(\left(1-\beta_{n}\right) x_{n}+\beta_{n} T x_{n}\right),
$$

where $T_{\lambda_{n}}=\left(I+r A_{F}\right)^{-1}$

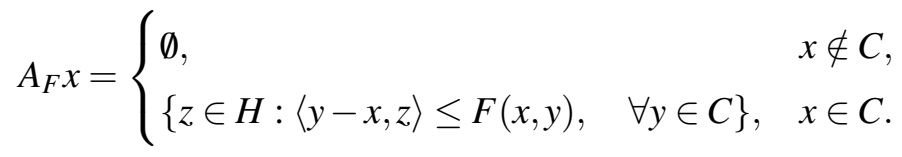

On the other hand, smooth constant $\Upsilon$ is $\frac{1}{\sqrt{2}}$ in the framework of Hilbert spaces. From Theorem 3.1, we can obtain the desired conclusion immediately. 


\section{Acknowledgements}

The author thanks the referees for their valuable comments and suggestions.

\section{REFERENCES}

[1] D.E. Alspach, A fixed point free nonexpansive map, 82 (1981), 423-424.

[2] F.E. Browder, Nonexpansive nonlinear operators in a Banach space, Proc. Natl. Acad. Sci. USA 54 (1965), $1041-1044$.

[3] W.A. Kirk, A fixed point theorem for mappings which do not increase distances, Amer. Math. Monthly 72 (1965), 10041006.

[4] S.Y. Cho, Generalized mixed equilibrium and fixed point problems in a Banach space, J. Nonlinear Sci. Appl. 9 (2016), 1083-1092.

[5] X. Qin, A. Petrusel, J.C. Yao, CQ iterative algorithms for fixed points of nonexpansive mappings and split feasibility problems in Hilbert spaces, J. Nonlinear Convex Anal. 19 (2018), 157-165.

[6] W. Takahashi, Weak and strong convergence theorems for families of nonlinear and nonself mappings in Hilbert spaces, J. Nonlinear Var. Anal. 1 (2017), 1-23.

[7] X. Qin, J.C. Yao, Weak convergence of a Mann-like algorithm for nonexpansive and accretive operators, J. Inequal. Appl. 2016 (2016), Article ID 232.

[8] X. Qin, S.Y. Cho, Convergence analysis of a monotone projection algorithm in reflexive Banach spaces. Acta Math. Sci. 37 (2017), 488-502.

[9] V. Barbu, Nonlinear Semigroups and Differential Equations in Banach Space, Noordhoff, Amsterdam, 1976.

[10] F.E. Browder, W.V. Petryshyn, Construction of fixed points of nonlinear mappings in Hilbert spaces, J. Math. Anal. Appl. 20 (1967), 197-228.

[11] F.E. Browder, Nonlinear mappings of nonexpansive and accretive type in Banach spaces, Bull. Amer. Math. Soc. 73 (1967), 875-882.

[12] T. Kato, Nonlinear semigroups and evolution equations, J. Math. Sco. Japan 19 (1967), 508-520.

[13] S.Y. Cho, B.A. Bin Dehaish, X. Qin, Weak convergence of a splitting algorithm in Hilbert spaces, J. Appl. Anal. Comput. 7 (2017), 427-438.

[14] S.Y. Cho, X. Qin, On the strong convergence of an iterative process for asymptotically strict pseudocontractions and equilibrium problems. Appl. Math. Comput. 235 (2014), 430-438.

[15] S.Y. Cho, X. Qin, L. Wang, Strong convergence of a splitting algorithm for treating monotone operators, Fixed Point Theory Appl. 2014 (2014), Article ID 94.

[16] X. Qin, S.Y. Cho, L. Wang, A regularization method for treating zero points of the sum of two monotone operators, Fixed Point Theory Appl. 2014 (2014), Article ID 75.

[17] M. Wen, C. Hu, J. Peng, Iterative approximation of zeros of accretive operators and of solutions to fixed point problems in q-uniformly smooth Banach spaces, J. Fixed Point Theory Appl. 19 (2017), 1439-1452.

[18] K. Aoyama, H. Iiduka, W. Takahashi, Weak convergence of an iterative sequence for accretive operators in Banach spaces, Fixed Point Theory Appl. 2006 (2006), Article ID 35390.

[19] S.S. Chang, C.F. Wen, J.C. Yao, Common zero point for a finite family of inclusion problems of accretive mappings in Banach spaces, Optimization, 67 (2018), 1183-1196.

[20] X. Qin, S.Y. Cho, L. Wang, Iterative algorithms with errors for zero points of m-accretive operators, Fixed Point Theory Appl. 2013 (2013), Article ID 148.

[21] X. Qin, J.C. Yao, Projection splitting algorithms for nonself operators, J. Nonlinear Convex Anal. 18 (2017), 925-935.

[22] L.C. Ceng, C.F. Wen, Y. Yao, Iteration approaches to hierarchical variational inequalities for infinite nonexpansive mappings and finding zero points of m-accretive operators, J. Nonlinear Var. Anal. 1 (2017), 213-235.

[23] I. Cioranescu, Geometry of Banach Spaces, Duality Mappings and Nonlinear Problems, Kluwer, Dordrecht, 1990.

[24] R.E. Bruck, The iterative solution of the equation $y \in x+T x$ for a monotone operator $T$ in Hilbert space, Bull. Amer. Math. Soc. 79 (1973), 1258-1262.

[25] H. Zhou, Convergence theorems for $\lambda$-strict pseudo-contractions in 2-uniformly smooth Banach spaces, Nonlinear Anal. 69 (2008), 3160-3173. 
[26] J.G. Falset, W. Kaczor, T. Kuczumow, S. Reich, Weak convergence theorems for asymptotically nonexpansive mappings and semigroups, Nonlinear Anal. 43 (2007), 377-401.

[27] X. Qin, S.Y. Cho, L. Wang, Strong convergence of an iterative algorithm involving nonlinear mappings of nonexpansive and accretive type, Optimization, doi: 10.1080/02331934.2018.1491973.

[28] E. Blum, W. Oettli, From optimization and variational inequalities to equilibrium problems, Math. Stud. 63 (1994), 123145.

[29] S. Takahashi, W. Takahashi, M. Toyoda, Strong convergence theorems for maximal monotone operators with nonlinear mappings in Hilbert spaces, J. Optim. Theory Appl. 147 (2010), 27-41. 